\title{
Role of DRAM1 in mitophagy contributes to preeclampsia regulation in mice
}

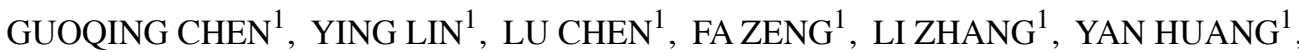 \\ PINGPING HUANG ${ }^{1}$, LINGLING LIAO ${ }^{1}$ and YUANLAN YU ${ }^{2}$ \\ ${ }^{1}$ Department of Obstetrics, Affiliated Shenzhen Maternity and Child Healthcare Hospital, \\ Southern Medical University, Shenzhen, Guangdong 518028; ${ }^{2}$ Department of Emergency, \\ Shenzhen Children's Hospital, Shenzhen, Guangdong 518026, P.R. China
}

Received January 10, 2019; Accepted June 2, 2020

DOI: $10.3892 / \mathrm{mmr} .2020 .11269$

\begin{abstract}
Preeclampsia (PE) is a complication during pregnancy that is diagnosed by a new onset of hypertension and proteinuria. Although the pathogenesis of PE is not fully understood, a growing body of evidence indicates that oxidative stress and mitochondrial dysfunction might contribute to the progression of PE. Therefore, the aim of the present study was to determine the role of mitophagy in mitochondrial dysfunction and oxidative stress in PE, and to evaluate the role of DNA damage-regulated autophagy modulator 1 (DRAM1) in the development of PE. First, a mouse model of PE induced by hypoxia-inducible factor $1 \alpha$ was established, and high levels of oxidative stress, apoptosis and mitochondrial dysfunction were found in the placentas of PE mice. Additionally, the placentas of PE mice exhibited decreased mitophagy and significantly decreased DRAM1 expression. To further explore the role of DRAM1 in mitophagy, DRAM1 was overexpressed in the placental tissues of PE mice, and this overexpression effectively improved the symptoms of PE mice and significantly reduced blood lipid and urine protein levels. DRAM1 overexpression also improved mitochondrial function and reduced oxidative stress in the placentas of PE mice. In addition, the overexpression of DRAM1 improved mitochondrial fusion and fission, and enhanced mitophagy. Altogether, these results indicated a key role for DRAM1 in mitophagy that contributed to the regulation of PE. To the best of the authors' knowledge, the present study provided the first evidence of a role for DRAM1 in PE, and offered novel insight into the pathophysiological mechanisms of PE.
\end{abstract}

Correspondence to: Dr Yuanlan Yu, Department of Emergency, Shenzhen Children's Hospital, 7019 Yitian Road, Shenzhen, Guangdong 518026, P.R. China

E-mail: yuyuanlansz@yeah.net

Key words: preeclampsia, oxidative stress, mitochondrial dysfunction, DNA damage-regulated autophagy modulator 1 , mitophagy

\section{Introduction}

Preeclampsia (PE) is characterized by hypertension with or without proteinuria after the 20th week of pregnancy, and is associated with endothelial dysfunction, oxidative stress, systemic vasoconstriction, inflammation, intrauterine growth restriction and multiorgan dysfunction $(1,2)$. The World Health Organization reports that $>60,000$ pregnant women worldwide die of preeclampsia each year (3). Women with PE and their offspring are also at increased risk of developing cardiovascular disease later in life (4).

Although the pathogenesis of PE remains unclear, increased oxidative stress in conjunction with antioxidative defence mechanisms may contribute to the progression of PE. Pregnancy itself increases susceptibility to oxidative stress, which could result in tissue damage (5). However, higher production of pro-oxidants and reactive oxygen species (ROS) is observed at the later stages of normal uncomplicated pregnancies and maintained through the accumulation of antioxidants, such as superoxide dismutase (SOD), glutathione (GSH), tocopherols, carotenoids and ascorbic acid (6). Nevertheless, the balance between pro-oxidants, such as malondialdehyde (MDA), and antioxidants, such as GSH and SOD, is disturbed in pregnancies with PE (7); this can lead to cellular dysfunction and death $(8)$. Moreover, previous studies $(9,10)$ have found that abnormal placentation and shallow trophoblast invasion stimulate placental oxidative stress, which leads to an intravascular inflammatory response and endothelial dysfunction (11). Although multiple sources of ROS have been identified in the placenta (12), such as the reduction of antioxidant activity and the lack of blood flow, few studies have examined the role of mitochondrial dysfunction and ROS in the pathology of PE. Mitochondrial dysfunction induces a reduction in oxygen consumption and an increase in superoxide production (13).

Mitochondria play an important role in ATP synthesis during aerobic respiration. During this process, ROS, such as the superoxide anion, hydroxyl radical, hydroperoxyl and hydrogen peroxide $\left(\mathrm{H}_{2} \mathrm{O}_{2}\right)$, are formed as metabolites of mitochondrial oxidative phosphorylation (14). Under conditions of oxidative stress, mitochondria can synthesize ROS at a level that extensively disrupts mitochondrial homeostasis, and alters the composition of lipids, proteins and nucleic acids (15). The 
resulting disruption in the composition of the mitochondrial membrane impairs the electrochemical potential of the membrane, which leads to mitochondrial dysfunction and apoptosis (16).

As mitochondrial damage can lead to apoptosis, the timely elimination of excess ROS is important for cell survival. Elevated levels of ROS trigger mitophagy, a cellular process in which lysosomes selectively scavenge for and eliminate damaged mitochondria (17). Under conditions of oxidative stress, mitophagy is upregulated to prevent the accumulation of dysfunctional mitochondria $(18,19)$. Thus, it was hypothesized that mitochondrial dysfunction regulated by mitophagy plays an important role in PE.

DNA damage-regulated autophagy modulator 1 (DRAM1) is an evolutionarily conserved transmembrane protein (20). This protein was previously identified as the first direct molecular link between the tumor suppressor p53 and autophagy (21). Since then, various roles for DRAM1 in several processes, including autophagy, cell death, immunity and cellular differentiation, have been described $(21,22)$. As stated above, elevated oxidative stress caused by mitochondrial dysfunction leads to apoptosis, and mitophagy plays an important role in the maintenance of mitochondrial function. However, neither mitochondrial dysfunction nor mitophagy have been examined in PE, and there is limited understanding regarding the contributions of mitochondrial dysfunction and mitophagy to the development of PE. Moreover, the causes of mitochondrial dysfunction and mitophagy in PE are unknown. Therefore, the aim of the present study was to evaluate the roles of mitochondrial dysfunction and ROS in the development of PE, and to determine the role of mitophagy in mitochondrial dysfunction in PE, in order to investigate whether DRAM1 plays a role in mitophagy and mitochondrial dysfunction in PE.

In the present study, it was demonstrated that oxidative stress was induced by mitochondrial dysfunction and decreased DRAM1 expression, and that increased mitophagy could cause mitochondrial dysfunction in the PE placenta. Additionally, it was demonstrated that the mitophagy dysfunction in PE might result from disturbances in mitochondrial fusion and fission due to a deficit in DRAM1. Altogether, the results of the present study indicated a key role for DRAM1 in mitophagy that contributed to PE. To the best of the authors' knowledge, this study provided the first evidence that DRAM1 plays a role in PE, and offers a new target for the treatment of PE and new insights into the pathophysiological mechanisms of PE.

\section{Materials and methods}

Animals. A total of 80 C57BL/6J wild-type (WT) mice (age, 2 months; weight, 30-40 g) were purchased from Guangdong Medical Laboratory Animal Center, and all the experiments were approved by the Ethics Committee of Shenzhen Hospital of Peking University (permit no. SYXK-2015-0106, 2019-078) and conducted in accordance with the ARRIVE guidelines on the Care and Use of Experimental Animals (23). Male and female animals were fed separately and housed in groups of four to five. All the mice were maintained under standard laboratory conditions with a temperature of $22 \pm 2^{\circ} \mathrm{C}$, $50 \pm 10 \%$ relative humidity and a 12 -h light/12-h dark cycle and provided with food and water ad libitum. The fertility cycle of the mice was controlled, and the animals were allowed to mate overnight when the females were in a pro-estrus state. On the following morning at 8:00 a.m., vaginal smears on glass slides were examined, and if spermatozoa were found, that day was designated the first day of pregnancy, embryonic day (E)0.5. Pregnant mice at E8 were randomly divided into three groups ( $\mathrm{n}=8 /$ group) and injected systemically via the tail vein with $100 \mu \mathrm{l}$ Ad-CMV-HIF (8x10 $0^{10}$ viral particles) encoding hypoxia-inducible factor $1 \alpha$ (Hif- $1 \alpha)$, Ad-CMV-GFP $\left(8 \times 10^{10}\right.$ viral particles) as a green fluorescent protein (GFP)-encoding vehicle (Veh-GFP) or saline solution [Shanghai OBiO Technology (Shanghai) Corp., Ltd.].

Blood pressure measurements. Systolic blood pressure of conscious mice was measured on E0.5, E8.5, E12.5, E16.5 and E19.5 of gestation using the tail cuff technique (24) (Shanghai Yuyan Instruments Co., Ltd.) The mice were prewarmed at $37^{\circ} \mathrm{C}$ for $30 \mathrm{~min}$ before the measurements were taken. The mean from five consecutive readings was recorded as the systolic blood pressure.

Urinary albumin and serum lipid measurements. Urine was collected on E16.5 and E19.5 of gestation for albumin measurements, and the urinary albumin was quantified using a bicinchoninic acid (BCA) protein assay with an Easy II Protein Quantitative kit (cat. no. DQ111-01; Beijing Transgen Biotech Co., Ltd.) according to the manufacturer's recommended protocol. In brief, urine was collected from each mouse, and the protein concentration was determined using a BCA protein assay. The absorbance at $595 \mathrm{~nm}$ was measured using a microplate reader, and BSA (Sigma-Aldrich; Merck $\mathrm{KGaA}$ ) was used as the standard.

Blood was collected on E19.5 of gestation for serum lipid measurements, and the level of serum lipids was quantified by ultraviolet-visible spectrophotometry using the following biochemical detection kits for blood lipids: Total cholesterol (TC; cat. no. BC1985; Beijing Solarbio Science \& Technology Co., Ltd.), triglyceride (TG; cat. no. BH017Z; Tellgen Corporation), low-density lipoprotein (LDL; cat. no. BH019Z; Tellgen Corporation) and high-density lipoprotein (HDL; cat. no. BH018Z; Tellgen Corporation). All assays were performed according to the manufacturer's protocols.

Tissue processing. For histological and biochemical analyses, the mice were euthanized, and their placentas and kidneys were rapidly removed. Some placentas were maintained at $80^{\circ} \mathrm{C}$ for biochemical analysis, and the remaining placentas and kidneys were fixed with $4 \%$ (vol/vol) paraformaldehyde overnight at $4^{\circ} \mathrm{C}$, dehydrated with gradient sucrose (20 and $\left.30 \%\right)$ for $24 \mathrm{~h}$ and embedded in optimal cutting temperature compound. The embedded tissue was sagittally cut into $30-\mu$ m-thick sections using a freezing-sliding microtome (Leica Microsystems $\mathrm{GmbH})$.

Histology. The obtained placental kidney tissue sections were washed with PBS and used for hematoxylin and eosin staining, which was performed according to the manufacturer's protocol (cat. no. G1120; Beijing Solarbio Science \& Technology Co., Ltd.). The sections were visualized using a light microscope (Leica DM4 M; magnification, x20). 
Table I. Antibody information.

\begin{tabular}{lllll}
\hline Antibody & Host species & Cat. no. & Dilution & Supplier \\
\hline Bax & Rabbit & ab32503 & $1: 2,000$ & Abcam \\
Bcl-2 & Rabbit & ab185002 & $1: 2,000$ & Abcam \\
COX IV & Rabbit & 4850 & $1: 1,000$ & Cell Signaling Technology, Inc. \\
PINK1 & Rabbit & ab216144 & $1: 1,000$ & Abcam \\
Parkin & Mouse & 4211 & $1: 1,000$ & Cell Signaling Technology, Inc. \\
DRAM1 & Rabbit & PA5-69473 & $1: 500$ & Invitrogen; Thermo Fisher Scientific, Inc. \\
Mfn1 & Rabbit & 14739 & $1: 1,000$ & Cell Signaling Technology, Inc. \\
Mfn2 & Rabbit & 11925 & $1: 1,000$ & Cell Signaling Technology, Inc. \\
OPA1 & Rabbit & ab157457 & $1: 1,000$ & Abcam \\
DRP1 & Rabbit & ab184247 & $1: 1,000$ & Abcam \\
GAPDH & Rabbit & ab181602 & $1: 2,000$ & Abcam \\
$\beta$-actin & Mouse & 3700 & $1: 2,000$ & Cell Signaling Technology, Inc. \\
\hline
\end{tabular}

DRAM1, DNA damage-regulated autophagy modulator 1; PINK1, phosphatase and tensin homolog-induced putative kinase 1; COX IV, cytochrome $c$ oxidase IV; Mfn1, mitofusin 1; Mfn2, mitofusin 2; OPA1, optic atrophy 1; DRP1, dynamin-related protein 1.

Oxidative stress measurements. To test the level of oxidative stress in the placenta, different kits were used. The activity of SOD in placental tissue was detected by nitro-blue tetrazolium (cat.no. S0107; Beyotime Institute of Biotechnology). The levels of MDA were tested by thiobarbituric acid (cat. no. BC0025; Beijing Solarbio Science \& Technology Co., Ltd.), and the levels of $\mathrm{H}_{2} \mathrm{O}_{2}$ were tested by titanium sulfate (cat. no. BC3595; Beijing Solarbio Science \& Technology Co., Ltd.). All assays were performed according to the manufacturer's protocol.

Western blotting. Placental samples were homogenized in RIPA lysis buffer (cat. no. P0013B; Beyotime Institute of Biotechnology), containing $1 \mathrm{mM}$ PMSF (Beyotime Institute of Biotechnology), a protease inhibitor cocktail and phosphatase inhibitors (Roche Diagnostics). The samples were centrifuged at $13,000 \times \mathrm{g}$ and $4^{\circ} \mathrm{C}$ for $30 \mathrm{~min}$, and the supernatants were collected. The protein concentration was measured with a BCA kit (cat. no. DQ111-01; Beijing Transgen Biotech Co., Ltd.). The protein samples $(20 \mu \mathrm{g})$ were loaded onto a $10 \%$ SDS-PAGE gel and then transferred onto $0.45-\mathrm{mm}$ polyvinylidene difluoride membranes (EMD Millipore) at $90 \mathrm{~mA}$ for 1-2 h. After blocking with 5\% nonfat milk for $1 \mathrm{~h}$ at $37^{\circ} \mathrm{C}$, the membrane was incubated with primary antibody (Table I) overnight at $4^{\circ} \mathrm{C}$ and horseradish peroxidase-conjugated secondary antibodies (anti-mouse and anti-rabbit; 1:500; cat. nos. 6919-100 and 6925-100; NeoBioscience) for $1 \mathrm{~h}$ at $37^{\circ} \mathrm{C}$. The immunoreactive bands were visualized with an enhanced chemiluminescence kit (cat. no. A38555; Thermo Fisher Scientific, Inc.) and scanned for densitometric analysis using an imaging system (Image Station 4000 M; Kodak) and Quantity One software v4.6.6 (Bio-Rad Laboratories, Inc.). GAPDH or $\beta$-actin were used as the loading control.

In utero electroporation (IUE). IUE was performed as previously described (25). Briefly, pregnant female PE mice (induced by Hif1- $\alpha$ ) at E14.5 were anesthetized by diluting ketamine $(100 \mathrm{mg} / \mathrm{kg})$ and xylazine $(10 \mathrm{mg} / \mathrm{kg})$ with $0.9 \%$ saline. The abdomen was cut, and the uterine horns were then carefully removed. Next, $\sim 3 \mu \mathrm{g}$ plasmid DNA (V5-DRAM1-pCAGEN-GFP or V5-pCAGEN-GFP; Biovector Science Lab, Inc.) was mixed with $1.5 \mu 10.025 \%$ Fast Green (cat. no. F7252; Sigma-Aldrich; Merck KGaA), which was pressure-injected into the junctional zone of the placenta by pulling the glass capillaries. Five pulses of current $(40 \mathrm{mV}$ for $40 \mathrm{msec}$ ) were injected into the placenta using an electroporator (BTX T830; BTX Molecular Delivery Systems). The uterus was moved into the peritoneal cavity, and the abdominal wall and skin were sutured. The transfection efficiency was determined using immunofluorescence and a confocal microscope (Nikon AR1; magnification, x20).

Statistical analysis. Data were analyzed using GraphPad Prism v 7.04 software (GraphPad Software, Inc.). In animal experiments, pregnant mice at E8 were randomly divided into three groups ( $n=8 /$ group) to determine the blood pressure and total urinary protein levels. To investigate TG, TC, LDL and HDL levels, 4 samples were chosen. All the data are expressed as mean \pm SEM. One-way ANOVA followed by Bonferroni's post hoc test was used for multiple comparisons among the WT (injected with saline only), Veh-GFP and Hif- $1 \alpha$ groups. Unpaired Student's t-test was used when comparing the control and DRAM1 groups. $\mathrm{P}<0.05$ was considered to indicate a statistically significant difference.

\section{Results}

Hif- $1 \alpha$ induces PE in mice. To effectively evaluate whether DRAM1 plays an important role in PE, a PE mouse model induced by Hif-1 $\alpha$ was established (26). The symptoms of the PE mouse model were then evaluated by examining pathological and physiological indicators associated with PE.

Hypertension is a defining feature of PE (27), and thus dynamic changes in blood pressure were examined in PE 
A

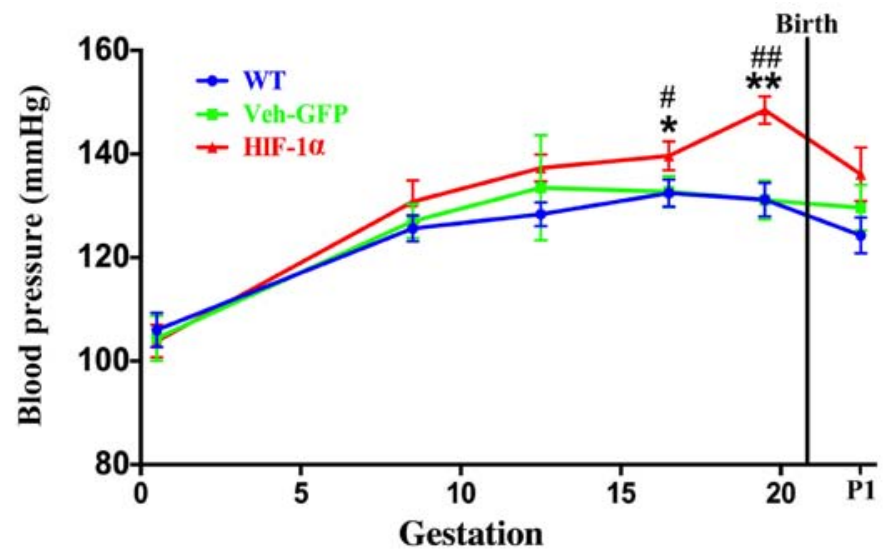

B
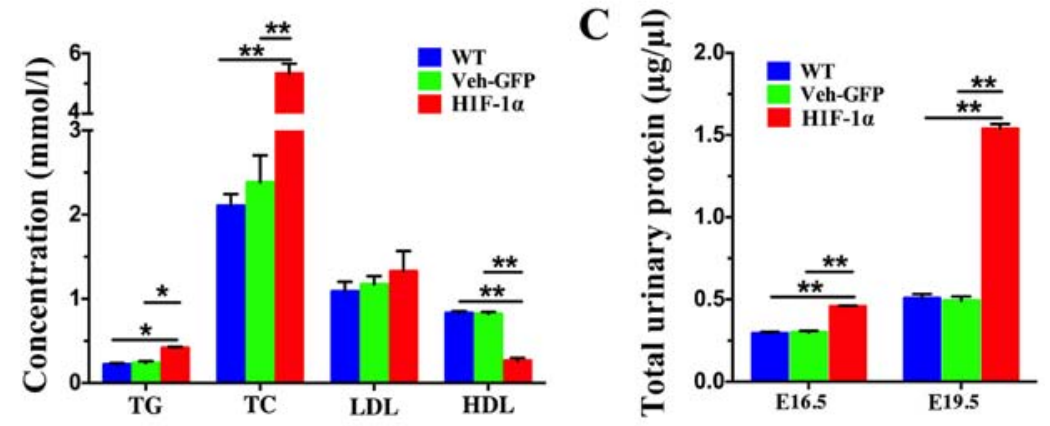

D

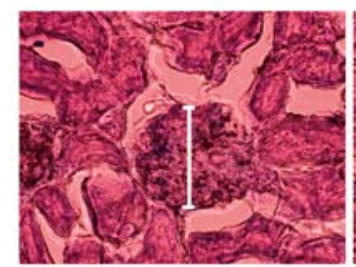

WT

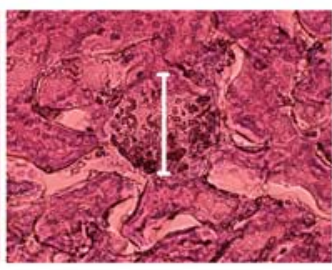

Veh-GFP

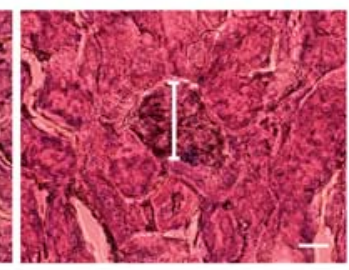

HIF-1a

Figure 1. Construction of a PE mouse model by Hif-1 $\alpha$ virus. PE mice exhibited elevated blood pressure, increased urinary protein levels and impaired renal function. (A) Blood pressure of the WT, Veh-GFP and Hif-1 $\alpha$ mice was measured throughout gestation at the indicated time points ( $\mathrm{n}=8$ per group). Data are presented as the mean \pm SEM from several mice used in each experiment. ${ }^{*} \mathrm{P}<0.05$ and ${ }^{* *} \mathrm{P}<0.01$ vs. Veh-GFP group; ${ }^{*} \mathrm{P}<0.05$ and ${ }^{\# \#} \mathrm{P}<0.01$ vs. WT group.(B) TG, TC, LDL and HDL levels in the serum of WT, Veh-GFP and Hif-1 $\alpha$ mice at E19.5 were measured ( $\mathrm{n}=4$ per group). Data are presented as the mean \pm SEM from several mice used in each experiment. ${ }^{*} \mathrm{P}<0.05,{ }^{* *} \mathrm{P}<0.01$. (C) Total urinary protein level in WT, Veh-GFP and Hif-1 $\alpha$ mice at E16.5 and E19.5 was determined using bicinchoninic acid protein assays $(\mathrm{n}=8)$. ${ }^{* * *} \mathrm{P}<0.01$. (D) Renal histology of WT, Veh-GFP and Hif-1 $\alpha$ mice at E19.5 demonstrated by hematoxylin and eosin staining. Scale bar, $50 \mu \mathrm{m}$. PE, preeclampsia; Hif-1 $\alpha$, hypoxia-inducible factor 1 $\alpha$; WT, wild-type; Veh-GFP, vehicle-green fluorescent protein; E, embryonic day; TG, triglyceride; TC, total cholesterol; LDL, low-density lipoprotein; HDL, high-density lipoprotein.

mice during the progression of pregnancy. The first measurement was taken at E0.5, and blood pressure was found to increase during pregnancy. On both E16.5 and E19.5, the blood pressure of the Hif-1 $\alpha$ group was significantly higher than that of the Veh-GFP and WT groups, and no significant difference was found between the Veh-GFP and WT groups (Fig. 1A). The blood pressure of the mice was also measured after delivery, which revealed that the blood pressure began to decrease following delivery, with no significant difference found between the three groups (Fig. 1A).

Dyslipidemia is closely related to the occurrence and development of PE during pregnancy (1). TG, TC, LDL and HDL levels were measured on E19.5. The results showed that the levels of TG and TC in the Hif-1 $\alpha$ group were significantly increased compared with those in the WT and Veh-GFP groups (Fig. 1B), with no significant difference detected between the WT and Veh-GFP groups. However, no significant change in the LDL content was found between the Hif- $1 \alpha$ group, and the WT and Veh-GFP groups (Fig. 1B). In addition, the HDL content of the Hif- $1 \alpha$ group was significantly decreased compared with that of the WT and Veh-GFP groups (Fig. 1B), and no significant difference was detected between the WT and Veh-GFP groups.

The presence of proteinuria, another defining feature of PE, was measured on E16.5 and E19.5. Urine protein content of the Hif-1 $\alpha$ group was significantly increased compared with that of the WT and Veh-GFP groups on both E16.5 and E19.5 (Fig. 1C). No significant differences were found between the WT and Veh-GFP groups on either E16.5 or E19.5 (Fig. 1C).

Kidney histology was assessed on E19.5, and it was found that the WT and Veh-GFP mice showed no glomerular changes compared with each other. Glomerular shrinkage was observed in the Hif- $1 \alpha$ mice relative to the WT and Veh-GFP mice (Fig. 1D). In addition, the Hif-1 $\alpha$ mice developed 
moderate swelling of glomerular endothelial cells and mesangial cells with limited occlusion of the glomerular capillary lumen (Fig. 1D).

The symptoms of hypertension, hyperlipidemia, high urine protein and renal dysfunction observed in the Hif- $1 \alpha$ mice were consistent with pathological observations in patients with PE. These results indicated successful establishment of a PE mouse model that could be used in subsequent experiments.

Excessive oxidative stress in the placentas of PE mice. The placenta is an important organ for the exchange of substances between the fetus and the mother. To assess whether the placental function of mice with PE was normal, the morphology and oxidative stress levels of these mice were evaluated. During the progression of gestation, the relative area of the junctional zone (JZ) decreases as the labyrinth expands in size (28). Firstly, morphological changes in the placentas of the mice were examined, and it was found that the $\mathrm{JZ}$ region of the Hif- $1 \alpha$ mice was increased compared with in the WT and Veh-GFP mice on E19.5 (Fig. 2A). To determine the level of oxidative stress in the placenta, the enzymatic activity of SOD, an important antioxidant enzyme, was measured. The results showed that the activity of SOD in the Hif-1 $\alpha$ group was significantly reduced compared with that of the WT and Veh-GFP groups, and no significant difference was found between the WT and Veh-GFP groups (Fig. 2B). The levels of MDA were also examined. MDA is a natural product of lipid oxidation in organisms and reflects levels of oxidative stress (24). It was found that the MDA content in the Hif- $1 \alpha$ group was significantly increased compared with in the WT and Veh-GFP groups, and no significant difference was detected between the WT and Veh-GFP groups (Fig. 2C). In addition, levels of $\mathrm{H}_{2} \mathrm{O}_{2}$, the most common active oxygen molecule in organisms, were measured. It was shown that $\mathrm{H}_{2} \mathrm{O}_{2}$ levels were increased significantly in the Hif- $1 \alpha$ group compared with the WT and Veh-GFP groups, and that no significant difference was found between the WT and Veh-GFP groups (Fig. 2C). These findings suggested the presence of excessive oxidative stress in the placentas of PE mice.

Mitochondrial dysfunction in the placenta of PE mice leads to apoptosis. High levels of oxidative stress usually trigger inflammation and apoptosis (29). There is a close relationship between oxidative stress and mitochondrial dysfunction, which can lead to the aggravation of oxidative stress (30). The levels of apoptosis and mitochondrial function were therefore examined.

Firstly, to investigate whether placental cells were growing normally, the expression of proteins associated with apoptosis was examined. Bax is one of the early-acting proapoptotic factors, and mainly exists in the cytoplasm and on the surface of mitochondria $(31,32)$. Bcl-2 is a classical gene that inhibits apoptosis (33); specifically, Bcl-2 is increased during the antioxidant status of cells, thereby protecting them from oxidative stress, which plays a certain role in reducing apoptosis, and it is mainly distributed in the mitochondrial membrane (31). Therefore, the levels of Bax and Bcl-2 and the ratio of Bax to Bcl-2 can be detected as a representation of the level of apoptosis.
Western blotting was used to assess the levels of Bax and Bcl-2. Bax protein expression in the Hif- $1 \alpha$ group was significantly increased compared with those in the WT and Veh-GFP groups, and no significant difference was found between the WT and Veh-GFP groups (Fig. 2E and F). In addition, no significant difference in the protein expression of Bcl-2 was detected between the WT, Veh-GFP or Hif-1 $\alpha$ groups (Fig. 2E and F). The Bax/Bcl-2 ratio found to be significantly increased in the Hif- $1 \alpha$ group compared with the WT and Veh-GFP groups, whilst there was no significant difference between the WT and Veh-GFP groups (Fig. 2E and F).

Mitochondria are the core of energy metabolism, and the growth of a fetus requires a large amount of ATP from the placenta (34). Therefore, mitochondrial dysfunction is an important factor that could affect PE. The maintenance of energy metabolism, the regulation of changes in mitochondrial function or adaptation to environmental stimulation, the generation of new mitochondria and the clearance of defective mitochondria are critical for the maintenance of mitochondrial health in eukaryotic cells $(35,36)$. In mitochondria, energy is mainly synthesized by the electron transport chain, and cytochrome $c$ oxidase IV (COX IV) plays an important role in this (37). Therefore, protein expression levels of COX IV in placental tissue were measured by western blotting. This showed that COX IV protein expression in the Hif-1 $\alpha$ group was significantly lower compared with in the WT and Veh-GFP groups (Fig. 2E and F), and that there was no significant difference between the WT and Veh-GFP groups. These results suggested that apoptosis induced by oxidative stress in the placentas of PE mice may be caused by mitochondrial dysfunction.

Decreased DRAM1 expression in the placentas of PE mice leads to mitophagy dysfunction. Mitophagy is the most important pathway for the clearance of damaged mitochondria. An important mitophagy pathway is the PTEN-induced putative kinase 1 (PINK1)/E3 ubiquitin ligase (Parkin) regulatory pathway (17). Protein aggregates and damaged organelles are ubiquitinated to initiate selective autophagy. For the initiation of mitophagy, phosphorylated PINK1 is ubiquitinated to activate Parkin; this process establishes a ubiquitin chain on outer mitochondrial membrane proteins, recruits autophagy receptors and triggers mitophagy $(38,39)$. Therefore, western blotting was performed to detect the protein expression of PINK1 and Parkin. This showed that the level of PINK1 in the Hif-1 $\alpha$ group was significantly lower compared with in the WT and Veh-GFP groups, and that there was no significant difference between the WT and Veh-GFP groups (Fig. 2G and H). Parkin protein expression in the Hif-1 $\alpha$ group was significantly decreased compared with that of the Veh-GFP group, and no significant difference was found between the WT and Veh-GFP groups (Fig. 2G and H).

The DRAM1 protein, which plays an important role in autophagy, was examined and it was discovered that DRAM1 protein expression in the Hif- $1 \alpha$ group was significantly decreased compared with in the WT and Veh-GFP groups, and no significant difference was detected between the WT and Veh-GFP groups (Fig. 2G and H).

These findings suggested that mitochondrial dysfunction may be due to the inability of damaged mitochondria 
A

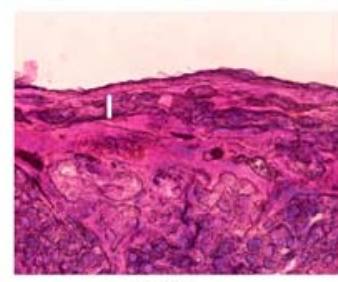

WT

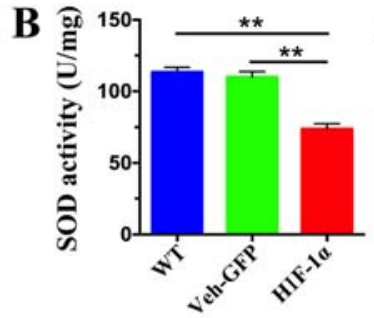

$\mathbf{E}$
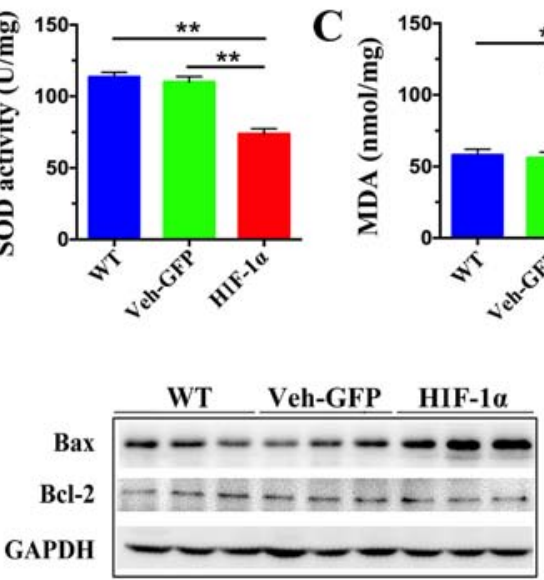

Veh-GFP

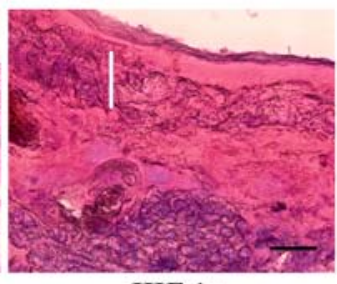

HIF-1 $\alpha$
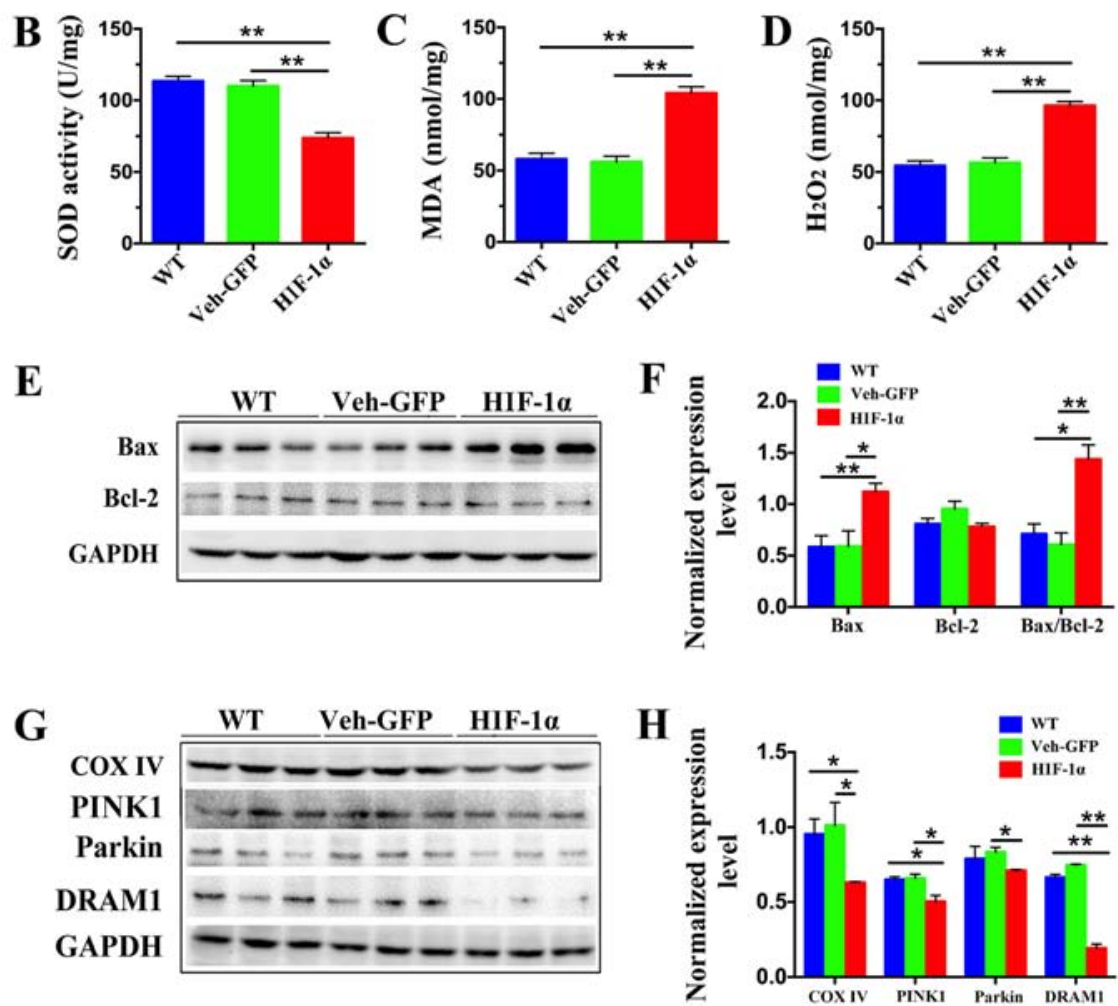

Figure 2. Levels of oxidative stress, apoptosis and mitochondrial autophagy are increased in the placenta of preeclampsia mice. (A) Morphological examination of mouse placenta at embryonic day 19.5 by hematoxylin and eosin staining revealed that the junctional zone (represented by the white line) of Hif-1 $\alpha$ mice was larger than those of WT and Veh-GFP mice. Scale bar, $100 \mu \mathrm{m}$. (B) Activity of SOD in placental tissue was detected by nitro-blue tetrazolium, (C) levels of MDA were measured using thiobarbituric acid and (D) levels of $\mathrm{H}_{2} \mathrm{O}_{2}$ were measured using titanium sulfate. ${ }^{* *} \mathrm{P}<0.01(\mathrm{n}=4)$. (E) Representative western blot images and (F) semi-quantification of Bax and Bcl-2 in the placenta of WT, Veh-GFP and Hif-1 $\alpha$ mice. Densitometry results were normalized against the levels of GAPDH. (G) Representative western blot images and $(\mathrm{H})$ semi-quantification of COX IV, PINK1, Parkin and DRAM1 in the placentas of WT, Veh-GFP and Hif-1 $\alpha$ mice. Densitometry results were normalized against the levels of GAPDH. Values are expressed as the mean $\pm \mathrm{SEM}(\mathrm{n}=3)$. ${ }^{*} \mathrm{P}<0.05,{ }^{* *} \mathrm{P}<0.01$. Hif- $1 \alpha$, hypoxia-inducible factor $1 \alpha$; WT, wild-type; Veh-GFP, vehicle-green fluorescent protein; SOD, superoxide dismutase; MDA, malondialdehyde; COX IV, cytochrome $c$ oxidase IV; PINK1, PTEN-induced putative kinase 1; DRAM1, DNA damage-regulated autophagy modulator 1.

to be cleared, and that the loss of DRAM1 may be related to mitophagy dysfunction.

Overexpression of DRAM1 significantly improves the symptoms of PE mice. To further investigate whether PE is affected by DRAM1, a DRAM1 overexpression plasmid was transferred into the trophoblasts of the placentas of PE mice on E14.5 via embryonic electroporation (Fig. 3A). Protein expression of DRAM1 was then tested by western blotting to verify the overexpression of DRAM1. It was demonstrated that the level of DRAM1 in the DRAM1 group was significantly increased compared with that in the control group (Fig. 3C and D). This result indicated that a model of DRAM1 overexpression was successfully established.

To evaluate the effect of DRAM1 overexpression on PE, the total amount of urine protein was measured in the mice. The results showed that there was no significant difference between the DRAM1 and control groups on E16.5, but that the level of urinary protein in the DRAM1 group was significantly decreased on E19.5 (Fig. 3B).

Moreover, the serum lipid levels of E19.5 mice were detected, which found no significant difference in the TG or LDL levels between the DRAM1 group and the control group (Fig. 3H). The TC content decreased significantly after DRAM1 overexpression, while the HDL content of the DRAM1 group was significantly increased compared with in the control group (Fig. 3H). Together, these results demonstrated that overexpression of DRAM1 effectively improved the symptoms of hyperlipidemia and high urine protein in PE mice.

DRAM1 overexpression effectively reduces the apoptosis of placental cells in PE mice. In addition, the level of oxidative stress was measured in placental tissues on E19.5; this showed that the level of SOD in the DRAM group was significantly 


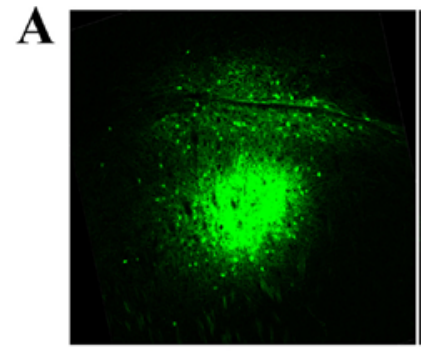

Control

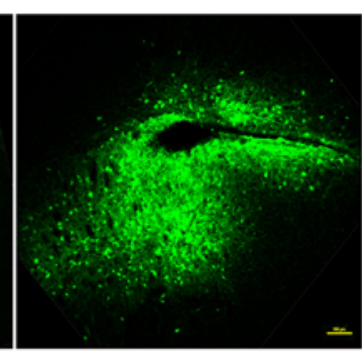

DRAM1
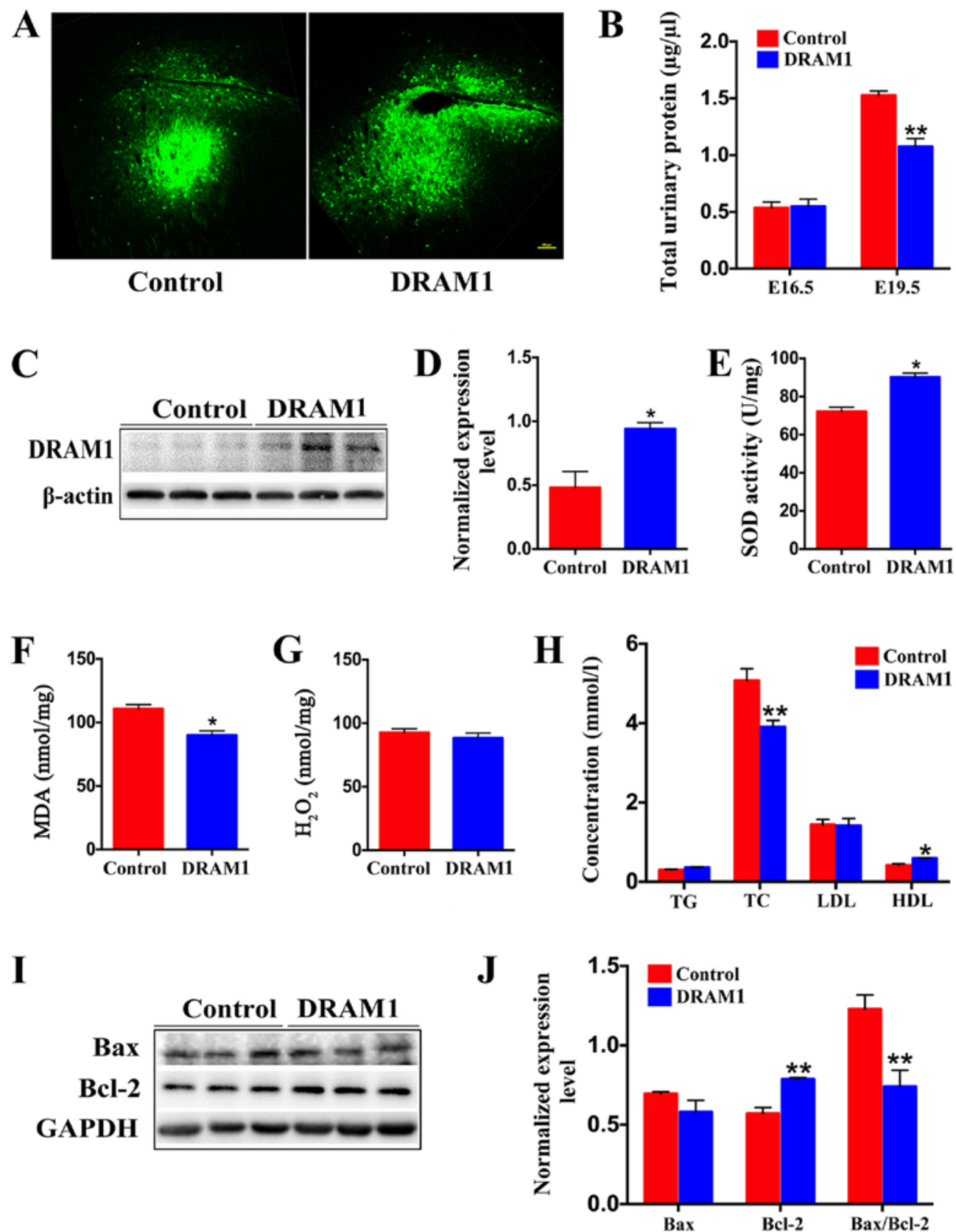

Figure 3. Overexpression of DRAM1 can effectively reduce apoptosis induced by oxidative stress and significantly reduce the urine protein and blood lipid levels in preeclampsia. (A) Representative images showing the overexpression of DRAM1 or control in the placenta of mice at E19.5. Scale bar, $100 \mu \mathrm{m}$. (B) Bicinchoninic acid protein assays were used to determine the total urinary protein level in the control and DRAM1 mice at E16.5 and E19.5 (n=6). (C) Representative western blot images and (D) semi-quantification of DRAM1 levels in the placenta of DRAM1-overexpressing or control mice at E19.5 $(n=3)$. Densitometry results were normalized against the levels of GAPDH. Levels of placental oxidative stress in DRAM1 mice was significantly decreased. (E) SOD in placental tissue was detected by nitro-blue tetrazolium, (F) levels of MDA were measured using thiobarbituric acid and (G) levels of $\mathrm{H}_{2} \mathrm{O}_{2}$ were measured using titanium sulfate $(\mathrm{n}=3)$. $(\mathrm{H}) \mathrm{TG}$, TC, LDL and HDL levels in the serum of control and DRAM1 mice at E19.5 (n=6). (I) Representative western blot images and $(\mathrm{J})$ semi-quantification of Bax and Bcl-2 in the placenta of control and DRAM1 mice. Densitometry results were normalized against the levels of GAPDH $(n=3)$. Data are presented as the mean \pm SEM. ${ }^{*} \mathrm{P}<0.05,{ }^{* *} \mathrm{P}<0.01$. DRAM1, DNA damage-regulated autophagy modulator 1 ; E, embryonic day; SOD, superoxide dismutase; MDA, malondialdehyde; TG, triglyceride; TC, total cholesterol; LDL, low-density lipoprotein; HDL, high-density lipoprotein.

higher compared with that in the control group (Fig. 3E). DRAM1 overexpression significantly decreased the level of MDA (Fig. 3F) and did not significantly change the level of $\mathrm{H}_{2} \mathrm{O}_{2}$ (Fig. 3G).

Apoptosis was subsequently studied in placental cells on E19.5. The DRAM1 group did not show a significant change in the level of Bax, while Bcl-2 protein expression was significantly increased compared with the control group (Fig. 3I and J). The Bax/Bcl-2 ratio was significantly decreased after DRAM1 overexpression (Fig. 3J). These results showed that the overexpression of DRAM1 effectively reduced oxidative stress-induced apoptosis.

The overexpression of DRAM1 promotes mitochondrial fusion, fission and mitophagy. To explore how DRAM1 attenuates the levels of oxidative stress and apoptosis in the placentas of PE mice, mitophagy and energy synthesis in the placenta were assessed after DRAM1 overexpression. Protein expression 
A

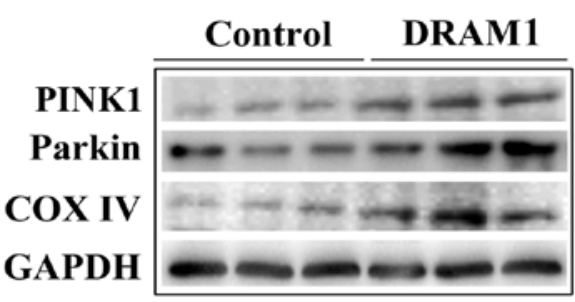

C

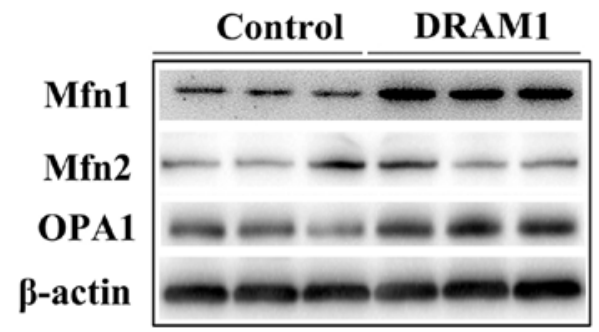

B

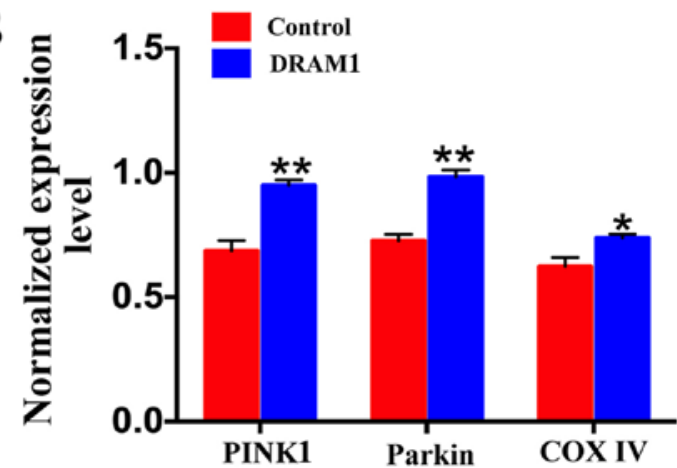

D

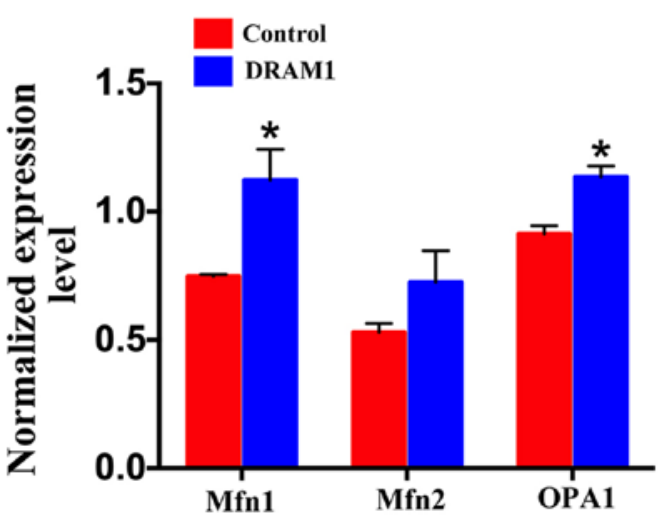

$\mathbf{E}$

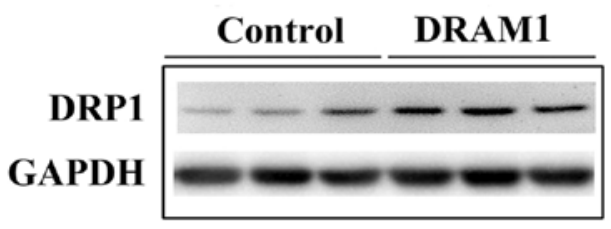

$\mathbf{F}$

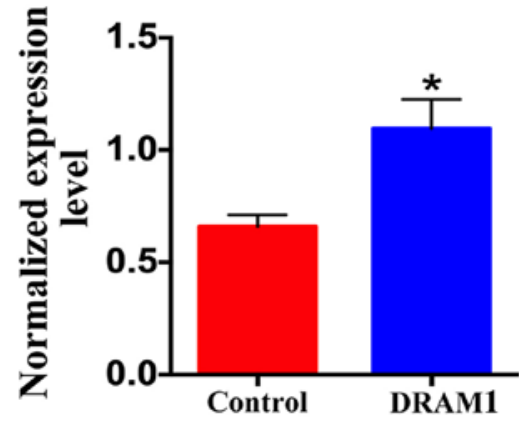

Figure 4. Overexpression of DRAM1 induces mitochondrial fusion and fission, and thus promotes mitophagy. (A) Representative western blot images and (B) semi-quantification of PINK1, Parkin and COX IV in the placenta of control and DRAM1 mice suggested that DRAM1 overexpression promotes mitophagy. (C) Representative western blot images and (D) semi-quantification of Mfn1, Mfn2 and OPA1 in the placenta of control and DRAM1 mice suggested that DRAM1 overexpression promotes mitochondrial fusion. (E) Representative western blot images and (F) semi-quantification of DRP1 in the placenta of control and DRAM1 mice suggested that DRAM1 overexpression promotes mitochondrial fission. Densitometry results were normalized against the levels of GAPDH or $\beta$-actin. Values are expressed as the mean \pm SEM $(n=3)$. ${ }^{*} \mathrm{P}<0.05,{ }^{* *} \mathrm{P}<0.01$. DRAM1, DNA damage-regulated autophagy modulator 1 ; PINK1, phosphatase and tensin homolog-induced putative kinase 1; COX IV, cytochrome $c$ oxidase IV; Mfn1, mitofusin 1; Mfn2, mitofusin 2; OPA1, optic atrophy 1; DRP1, dynamin-related protein 1.

of PINK1 and Parkin in the DRAM1 group was significantly increased compared with in the control group (Fig. 4A and B). These results indicated that the overexpression of DRAM1 effectively promoted the occurrence of mitophagy. Overexpression of DRAM1 also significantly promoted the expression of COX IV (Fig. 4A and B). Together, these results indicate that the overexpression of DRAM1 can promote mitophagy, clear damaged mitochondria and improve mitochondrial function.

The mitochondrial kinetic balance is critical for the regulation of mitochondrial function, and the maintenance of both energy metabolism and normal function through continuous fission and fusion (40). Therefore, changes in proteins associated with mitochondrial fission and fusion were examined. Mitochondria have a double-layer membrane structure, and the fusion of mitochondria is performed by optic atrophy 1 (OPA1) and mitofusin (Mfn)1/2 (41). The fusion of the outer mitochondrial membrane is mediated by Mfn $1 / 2$, and OPA 1 mediates that of the inner membrane. Protein expression of Mfn1/2 in the placenta was measured using western blotting. This showed that Mfn1 expression was significantly increased (Fig. 4C and D), and that Mfn2 expression was not significantly altered (Fig. 4C and D), in the DRAM1 group compared with that of the control group. OPA1 protein levels in the mouse placenta was significantly increased in the DRAM1 group compared with the control group (Fig. 4C and D). The expression levels of DRP1 were also significantly increased 
in the DRAM1 group compared with the control group (Fig. 4E and F). These results indicated that DRAM1 can promote both the fusion and fission of mitochondria.

\section{Discussion}

Mitochondrial dysfunction and oxidative stress are associated with PE, but thus far their levels have not been measured in a mouse model of PE. However, such an investigation is important for obtaining an improved understanding of the contribution of mitochondrial dysfunction and oxidative stress to the function of the placenta in PE. The present study revealed several novel findings: i) The blood pressure of PE mice increased gradually during gestation and recovered gradually after the end of gestation; ii) PE mice exhibited high levels of oxidative stress in the placenta, leading to apoptosis; iii) the placentas of PE mice exhibited mitochondrial dysfunction, weaker mitophagy and low levels of DRAM1; iv) DRAM1 overexpression effectively reduced both the urine protein content and serum lipid levels in the placentas of PE mice; v) DRAM1 overexpression significantly reduced the level of oxidative stress, improved mitochondrial function and enhanced the level of mitophagy in the placentas of PE mice; and vi) DRAM1 improved mitophagy by promoting mitochondrial fusion and fission, thereby improving mitochondrial function. These results indicated that increased DRAM1 in the placenta contributed to improved mitochondrial dysfunction and decreased levels of oxidative stress in PE.

Although ROS production is known to be induced by the ischemic placenta in PE, ROS are also produced by systemic blood vessels during the second phase of PE onset (12). Certain studies have revealed that placental homogenates derived from patients with $\mathrm{PE}$ show $39 \%$ higher $\mathrm{H}_{2} \mathrm{O}_{2}$ production than those derived from healthy pregnant women $(3,42)$. The present study also found a significant increase in the level of $\mathrm{H}_{2} \mathrm{O}_{2}$ in a mouse model of PE induced by Hif- $1 \alpha$. However, the content of $\mathrm{H}_{2} \mathrm{O}_{2}$ in the placenta decreased after DRAM1 overexpression in PE mice. Furthermore, SOD is an enzyme that catalyzes the scavenging of superoxide radicals, which are constitutively expressed in the mitochondria and cytoplasm (43). SOD is increased during normal pregnancy, but the activity and mRNA expression of SOD in placentas derived from patients with $\mathrm{PE}$ are decreased, which might result in increased oxidative stress in the placentas of patients with PE $(44,45)$. It has been reported that SOD is decreased in erythrocytes derived from patients with PE. The present study also found a significant decrease in the activity of SOD in the placentas of PE mice, and the activity of SOD was increased significantly after overexpression of DRAM1.

Moreover, increased ROS concentrations in patients with PE have been confirmed by the measurement of increased levels of MDA, which is an index of lipid peroxidation (46). Consistently, the present study also found that the expression of MDA in the placentas of PE mice was significantly increased, and that the MDA content decreased significantly after DRAM1 overexpression. In addition, lipid peroxidation is closely related to heat-shock protein 70 (HSP70) (44). Previously, the HSP70 level in the peripheral blood was shown to be significantly higher in both the fetal and maternal circulation in PE (47). The present study also found that the serum TC and TG levels in the placentas of PE mice were significantly increased, and that these levels gradually decreased after DRAM1 overexpression.

Oxidative stress is the result of ROS content exceeding the available antioxidant activity, and is one of the risk factors for the development of PE through vascular dysfunction (45). Poor invasion of cytotrophoblasts into the uterine myometrium and disturbed spiral artery remodeling play important roles in the pathophysiology of $\mathrm{PE}$, which results in placental hypoxia $(48,49)$. At present, termination of pregnancy is the only method for avoiding maternal eclampsia and fetal distress in PE (50). Supplementation with numerous antioxidants might prevent or repair the occurrence of PE (51). The results of the present study indicated that DRAM1 overexpression can effectively reduce oxidative stress levels in the placentas of $\mathrm{PE}$ mice, which could be useful in developing a treatment for PE.

Over the past 20 years, $>800$ peer-reviewed publications have validated the hypothesis that oxidative damage is involved in the pathophysiology of PE; however, current antioxidant interventions are not clinically effective (52). One possible explanation is that these antioxidant regimens fail to reach the mitochondria in the cell and thus fail to improve the pathology of oxidative damage. Great advances have been made in mitochondrial pharmacology due to the development of a number of different pharmacological strategies for addressing mitochondrial dysfunction (53). Mitochondrial dysfunction is a pathogenic mediator of oxidative stress in PE, as demonstrated by the observation that the placentas of women with pregnancies complicated by PE exhibit increased mitochondrial lipid peroxidation and enhanced susceptibility of mitochondria to oxidation $(54,55)$. The present study also found that COX IV levels were significantly decreased in the placentas of PE mice.

There are a number of different indicators of disrupted mitochondrial function, such as altered oxygen consumption, decreased ATP production, increased ROS production and mtDNA damage (56). Mitochondrial damage is one of the most important factors that leads to mitochondrial dysfunction (43). Therefore, the prevention of mitochondrial damage is very important, and several quality control mechanisms have evolved to prevent mitochondrial damage and preserve a population of healthy mitochondria. The selective autophagy of mitochondria, which is known as mitophagy, is an important mitochondrial quality control mechanism that eliminates damaged mitochondria (43). The elimination of damaged mitochondria in mammals is mediated by a pathway involving PINK1 and Parkin (18). PINK1 and Parkin accumulate on damaged mitochondria, promote their segregation from the mitochondrial network and target these organelles for autophagic degradation through a process that requires the Parkin-dependent ubiquitination of mitochondrial proteins $(57,58)$. In the present study, significant decreases in the levels of PINK1 and Parkin in the placental tissues of PE mice were observed, suggesting that the mitochondrial dysfunction in PE is likely due to a decreased level of mitophagy. DRAM1 overexpression significantly increased the levels of PINK1 and Parkin in the placentas of PE mice, which indicated that mitophagy was effectively activated. COX IV protein expression was also increased, which indicated an effective improvement in mitochondrial function. 
How DRAM1 could improve mitophagy and thus promote recovery of mitochondrial function is important to explore. Mitochondrial dynamics are regulated via mitochondrial biogenesis, and continuous cycles of fission and fusion (40). These processes are hypothesized to target damaged/depolarized mitophagy (38). Mitochondrial fission and fusion are regulated by members of a family of conserved large GTPases that were initially identified in yeast (41). The dynamin-like GTPase dynamin-related protein 1 (DRP1) mediates fission by forming a multimeric complex that wraps around the outer membrane of mitochondrial tubules and exerts a mechanical force to produce membrane scission (17). In contrast to fission, during which mitochondria are divided using only an outer membrane apparatus, two distinct machineries, Mfn1/2 and OPA1, are required for fusion of the outer and inner membranes (41). In the present study, OPA1 and Mfn1/2 protein expression was measured, and it was found that mitochondrial fusion was significantly increased after DRAM1 overexpression. These findings are consistent with the findings reported by Zhang et al (59), who found that Mfn2 is downregulated in PE placentas. In addition, Zhang et al (59) demonstrated that ATP is significantly reduced after knockout of the Mfn2 gene in trophoblast cells. Therefore, the findings of the present study suggested that DRAM1 can improve mitochondrial dysfunction by promoting mitochondrial fusion in PE. Alternatively, during fission, unhealthy, nonfunctional mitochondrial fragments that lack transmembrane potentials are discarded and targeted for degradation via mitophagy (60). Wakabayashi et al (61) demonstrated that DRP1 $1^{-/-}$mice exhibit embryonic lethality due to a deficiency in the formation of trophoblast giant cells and consequent placental dysfunction, which underscores the requirement of mitochondrial fission for proper placental and embryonic development. Hence, DRAM1 promotes mitochondrial fission to accelerate mitophagy for the clearance of damaged mitochondria, which is significant for the maintenance of mitochondrial function.

As previously reported, the expression of DRAM1 could induce apoptosis in an autophagy dependent or independent manner $(21,62)$. However, the mechanism via which DRAM1 induces apoptosis or autophagic apoptosis remains unclear. In addition, it has been reported that inflammation-associated apoptosis is regulated by Bcl-2 and $\operatorname{Bax}(63,64)$, and that decreased invasion and migration ability of trophoblast cells induced by cell apoptosis in the placenta may be the major causes of PE $(65,66)$. Therefore, the specific mechanism of DRAM1 and the relationship of between inflammation and Bcl-2 and Bax requires further investigation.

In conclusion, to the authors' best knowledge, the present study provided the first demonstration that DRAM1 reduces oxidative stress-induced apoptosis by improving mitochondrial dysfunction, enhancing mitophagy clearance through mitochondrial fission and fusion, and rescuing urinary protein and serum lipid levels in the placentas of PE mice. These data shed light on a novel mechanism underlying the therapeutic effect of DRAM1 in PE.

\section{Acknowledgements}

The authors are grateful to Professor Yao-ting Gui and Professor Guang-hui Cui of Shenzhen Hospital of Peking
University for their assistance with the technical and animal experiments.

\section{Funding}

The present study was supported by the Medical Science and Technology Research Fund Project of Guangdong (grant no. A2019363), the National Science Foundation of China (grant nos. 81260385 and 81360383), the Clinical Research Project of Shenzhen Municipal Health Commission (grant no. SZLY2017017) and the Doctoral Project of Shenzhen Maternal and Child Health Hospital (grant no. FYA2018005).

\section{Availability of data and materials}

The datasets used and/or analyzed during the current study are available from the corresponding author on reasonable request.

\section{Authors' contributions}

GQC contributed to the acquisition and interpretation of the data, performed the experiments and statistical analyses, and participated in the drafting of the manuscript. YL, LC and FZ analyzed and interpreted the data, and LZ, YH, PPH and LL performed the statistical analyses. YL and LL participated in the drafting of the manuscript. YLY designed the study, performed the experiments, analyzed and interpreted the data, drafted the manuscript, procured funding and supervised the study. All authors read and approved the final manuscript.

\section{Ethics approval and consent to participate}

All animal experiments and procedures were approved by the Ethics Committee of Shenzhen Hospital of Peking University (permit no. SYXK-2015-0106, 2019-078).

\section{Patient consent for publication}

Not applicable.

\section{Competing interests}

The authors declare that they have no competing interests.

\section{References}

1. Reijnders D, Olson KN, Liu CC, Beckers KF, Ghosh S, Redman LM and Sones JL: Dyslipidemia and the role of adipose tissue in early pregnancy in the BPH/5 mouse model for preeclampsia. Am J Physiol Regul Integr Comp Physiol 317: R49-R58, 2019.

2. Kay VR, Ratsep MT,Figueiro-FilhoEA and Croy BA:Preeclampsia may influence offspring neuroanatomy and cognitive function: A role for placental growth factor? Biol Reprod 101: 271-283, 2019.

3. Maged AM, Shoab AY and Dieb AS: Antepartum and postpartum uterine artery impedance in women with pre-eclampsia: A case control study. J Obstet Gynaecol 39: 633-638, 2019.

4. Benkő Z, Chaveeva P, de Paco Matallana C, Zingler E, Wright A, Wright D and Nicolaides KH: Validation of competing-risks model in screening for pre-eclampsia in twin pregnancy by maternal factors. Ultrasound Obstet Gynecol 53: 649-654, 2019.

5. Shin EK, Kang HY, Yang H, Jung EM and Jeung EB: The regulation of fatty acid oxidation in human preeclampsia. Reprod Sci 23: 1422-1433, 2016 
6. Sun MN, Yang Z and Ma RQ: Interaction of fatty acid oxidation with oxidative stress in preeclampsia-like mouse model at multiple stages of gestation. Zhonghua Yi Xue Za Zhi 91: 2343-2347, 2011 (In Chinese).

7. Huai J, Yang Z, Yi YH and Wang GJ: Role of mammalian target of rapamycin signaling pathway in regulation of fatty acid oxidation in a preeclampsia-like mouse model treated with pravastatin Chin Med J (Engl) 132: 671-679, 2019.

8. Zárate A, Saucedo R, Valencia J, Manuel L and Hernández M: Early disturbed placental ischemia and hypoxia creates immune alteration and vascular disorder causing preeclampsia. Arch Med Res 45: 519-524, 2014

9. Schoots MH, Gordijn SJ, Scherjon SA, van Goor $\mathrm{H}$ and Hillebrands JL: Oxidative stress in placental pathology. Placenta 69: 153-161, 2018.

10. Aouache R, Biquard L, Vaiman D and Miralles F: Oxidative Stress in Preeclampsia and Placental Diseases. Int J Mol Sci 19: 1496,2018

11. Lecarpentier E and Tsatsaris V: Angiogenic balance (sFlt-1/PlGF) and preeclampsia. Ann Endocrinol (Paris) 77 97-100, 2016

12. Taysi S, Tascan AS, Ugur MG and Demir M: Radicals, Oxidative/Nitrosative stress and preeclampsia. Mini Rev Med Chem 19: 178-193, 2019.

13. Saito $S$ and Nakashima A: A review of the mechanism for poor placentation in early-onset preeclampsia: The role of autophagy in trophoblast invasion and vascular remodeling. J Reprod Immunol 101-102: 80-88, 2014.

14. Klingenberg M: The ADP-ATP translocation in mitochondria, a membrane potential controlled transport. J Membr Biol 56 97-105, 1980

15. Kalyanaraman B, Cheng G, Hardy M, Ouari O, Bennett B and Zielonka J: Teaching the basics of reactive oxygen species and their relevance to cancer biology: Mitochondrial reactive oxygen species detection, redox signaling, and targeted therapies. Redox Biol 15: 347-362, 2018.

16. Samartsev VN, Vedernikov AA, Khoroshavina EI and Dubinin MV: Comparative study of free oxidation and ATP synthesis in mitochondria in the liver of different animal species. J Evol Biochem Phys 53: 245-247, 2017.

17. Kubli DA, Zhang X, Lee Y, Hanna RA, Quinsay MN, Nguyen CK, Jimenez R, Petrosyan S, Murphy AN and Gustafsson AB: Parkin protein deficiency exacerbates cardiac injury and reduces survival following myocardial infarction. J Biol Chem 288 : 915-926, 2013.

18. Tusi SK, Khodagholi F and Sanati MH: Mitophagy pathway is induced by alginate oligosaccharide in PC12 cells exposed to oxidative stress. Alzheimers Dement 9: 307, 2013

19. Oyewole AO and Birch-Machin MA: Mitochondria-targeted antioxidants. FASEB J 29: 4766-4771, 2015.

20. Zhang XD, Qi L, Wu JC and Qin ZH: DRAM1 regulates autophagy flux through lysosomes. PLoS One 8: e63245, 2013.

21. Liu D, Li R, Guo X, Pang L, Zang Y, Liu K and Chen D: DNA damage regulated autophagy modulator 1 recovers the function of apoptosis-stimulating of p53 protein 2 on inducing apoptotic cell death in Huh7.5 cells. Oncol Lett 15: 9333-9338, 2018.

22. Nagata M, Arakawa S, Yamaguchi H, Torii S, Endo H, Tsujioka M, Honda S, Nishida Y, Konishi A and Shimizu S: Dram1 regulates DNA damage-induced alternative autophagy. Cell Stress 2: 55-65, 2018.

23. Cho A and Seung-Hyeok S: Ethical Guidelines for Use of Experimental Animals in Biomedical Research. J Bacteriol Virol 43: 18-26, 2013

24. Wilde E, Aubdool AA, Thakore P, Baldissera L Jr, Alawi KM, Keeble J, Nandi $M$ and Brain SD: Tail-Cuff technique and its influence on central blood pressure in the mouse. J Am Heart Assoc 6: e005204, 2017.

25. Saito T: In vivo electroporation in the embryonic mouse central nervous system. Nat Protoc 1: 1552-1558, 2006.

26. Albers RE, Kaufman MR, Natale BV, Keoni C, Kulkarni-Datar K, Min S, Williams CR, Natale DRC and Brown TL: Trophoblast-Specific expression of Hif- $1 \alpha$ results in Preeclampsia-Like symptoms and fetal growth restriction. Sci Rep 9: 2742, 2019.

27. Iriyama T, Wang W, Parchim NF, Song A, Blackwell SC, Sibai BM, Kellems RE and Xia Y: Hypoxia-independent upregulation of placental hypoxia inducible factor- $1 \alpha$ gene expression contributes to the pathogenesis of preeclampsia. Hypertension 65: $1307-1315,2015$
28. Zadora J, Singh M, Herse F, Przybyl L, Haase N, Golic M, Yung HW, Huppertz B, Cartwright JE, Whitley G, et al: Disturbed placental imprinting in preeclampsia leads to altered expression of DLX5, a Human-Specific early trophoblast marker. Circulation 136: 1824-1839, 2017.

29. Samimi M, Pourhanifeh MH, Mehdizadehkashi A, Eftekhar T and Asemi Z: The role of inflammation, oxidative stress, angiogenesis, and apoptosis in the pathophysiology of endometriosis: Basic science and new insights based on gene expression. J Cell Physiol 234: 19384-19392, 2019.

30. Wu MY, Yiang GT, Lai TT and Li CJ: The oxidative stress and mitochondrial dysfunction during the pathogenesis of diabetic retinopathy. Oxid Med Cell Longev 2018: 3420187, 2018.

31. Hassan M, Watari H, AbuAlmaaty A, Ohba Y and Sakuragi N: Apoptosis and molecular targeting therapy in cancer. Biomed Res Int 2014: 150845, 2014.

32. Demendi C, Börzsönyi B, Végh V, Nagy ZB, Rigó J Jr, Pajor A and Joó JG: Gene expression patterns of the Bcl-2 and Bax genes in preterm birth. Acta Obstet Gynecol Scand 91: 1212-1217, 2012.

33. Irani RA, Zhang Y, Blackwell SC, Zhou CC, Ramin SM, Kellems RE and Xia Y: The detrimental role of angiotensin receptor agonistic autoantibodies in intrauterine growth restriction seen in preeclampsia. J Exp Med 206: 2809-2822, 2009.

34. Han Y, Yang Z, Ding X, Yu H and Yi Y: Expression of long chain fatty acid oxidase in maternal and fetal tissues in preeclampsia-like mouse model in mid-gestation. Zhonghua Yi Xue Za Zhi 95: 26-29, 2015 (In Chinese).

35. Whitley BN, Engelhart EA and Hoppins S: Mitochondrial dynamics and their potential as a therapeutic target. Mitochondrion 49: 269-283, 2019.

36. Koch RE, Josefson CC and Hill GE: Mitochondrial function, ornamentation, and immunocompetence. Biol Rev Camb Philos Soc 92: 1459-1474, 2017.

37. Adam-Vizi V: Production of reactive oxygen species in brain mitochondria: Contribution by electron transport chain and non-electron transport chain sources. Antioxid Redox Signal 7: 1140-1149, 2005

38. Yoo SM and Jung YK: A molecular approach to mitophagy and mitochondrial dynamics. Mol Cells 41: 18-26, 2018.

39. Villa E, Marchetti S and Ricci JE: No Parkin Zone: Mitophagy without Parkin. Trends Cell Biol 28: 882-895, 2018.

40. Guzmán-Lastra F, Kaiser A and Löwen H: Fission and fusion scenarios for magnetic microswimmer clusters. Nat Commun 7 : 13519, 2016.

41. Kanki T, Klionsky DJ and Okamoto K: Mitochondria autophagy in yeast. Antioxid Redox Signal 14: 1989-2001, 2011.

42. Bartha JL, Visiedo F, Fernández-Deudero A, Bugatto F and Perdomo G: Decreased mitochondrial fatty acid oxidation in placentas from women with preeclampsia. Placenta 33: 132-134, 2012.

43. Beretta S, Sala G, Mattavelli L, Ceresa C, Casciati A, Ferri A, Carrì MT and Ferrarese C: Mitochondrial dysfunction due to mutant copper/zinc superoxide dismutase associated with amyotrophic lateral sclerosis is reversed by $\mathrm{N}$-acetylcysteine. Neurobiol Dis 13: 213-221, 2003

44. Ilhan N, Ilhan N and Simsek M: The changes of trace elements, malondialdehyde levels and superoxide dismutase activities in pregnancy with or without preeclampsia. Clin Biochem 35: 393-397, 2002.

45. Xuan RR, Niu TT and Chen HM: Astaxanthin blocks preeclampsia progression by suppressing oxidative stress and inflammation. Mol Med Rep 14: 2697-2704, 2016.

46. Feng YL, Yin YX, Ding J, Yuan H, Yang L, Xu JJ and Hu LQ: Alpha-1-antitrypsin suppresses oxidative stress in preeclampsia by inhibiting the p38MAPK signaling pathway: An in vivo and in vitro study. PLoS One 12: e0173711, 2017.

47. Fekete A, Vér A, Bögi K, Treszl A and Rigó J Jr: Is preeclampsia associated with higher frequency of HSP70 gene polymorphisms? Eur J Obstet Gynecol Reprod Biol 126: 197-200, 2006.

48. Yan JY and Xu X: Relationships between concentrations of free fatty acid in serum and oxidative-damage levels in placental mitochondria and preeclampsia. Zhonghua Fu Chan $\mathrm{Ke} \mathrm{Za}$ Zhi 47: 412-417, 2012 (In Chinese)

49. Hilali N, Kocyigit A, Demir M, Camuzcuoglu A, Incebiyik A, Camuzcuoglu H, Vural $M$ and Taskin A: DNA damage and oxidative stress in patients with mild preeclampsia and offspring. Eur J Obstet Gynecol Reprod Biol 170: 377-380, 2013. 
50. Salomon C, Yee SW, Mitchell MD and Rice GE: The possible role of extravillous trophoblast-derived exosomes on the uterine spiral arterial remodeling under both normal and pathological conditions. Biomed Res Int 2014: 693157, 2014.

51. Salles AM, Galvao TF, Silva MT, Motta LCD and Pereira MG: Antioxidants for preventing preeclampsia: A systematic review. ScientificWorldJournal 2012: 243476, 2012.

52. Thomas T, Jophy R, Mhaskar A and Misquith D: Are we increasing serious maternal morbidity by postponing termination of pregnancy in severe pre-eclampsia/eclampsia? J Obstet Gynaecol 25: 347-351, 2005.

53. Vishnyakova PA, Volodina MA, Tarasova NV, Marey MV, Tsvirkun DV, Vavina OV, Khodzhaeva ZS, Kan NE, Menon R, Vysokikh MY and Sukhikh GT: Mitochondrial role in adaptive response to stress conditions in preeclampsia. Sci Rep 6: 32410, 2016.

54. Zsengellér ZK, Rajakumar A, Hunter JT, Salahuddin S, Rana S, Stillman IE and Ananth Karumanchi S: Trophoblast mitochondrial function is impaired in preeclampsia and correlates negatively with the expression of soluble fms-like tyrosine kinase 1. Pregnancy Hypertens 6: 313-319, 2016.

55. Abad C, Vargas FR, Zoltan T, Proverbio T, Piñero S, Proverbio F and Marín R: Magnesium sulfate affords protection against oxidative damage during severe preeclampsia. Placenta 36: $179-185,2015$

56. Kumar A, Davuluri G, Welch N, Kim A, Gangadhariah M, Allawy A, Priyadarshini A, McMullen MR, Sandlers Y, Willard B, et al: Oxidative stress mediates ethanol-induced skeletal muscle mitochondrial dysfunction and dysregulated protein synthesis and autophagy. Free Radic Biol Med 145: 284-299, 2019.

57. Nakashima A, Tsuda S, Kusabiraki T, Aoki A, Ushijima A, Shima T, Cheng SB, Sharma S and Saito S: Current understanding of autophagy in pregnancy. Int J Mol Sci 20: 2342, 2019.

58. Ausman J, Abbade J, Ermini L, Farrell A, Tagliaferro A, Post M and Caniggia I: Ceramide-induced BOK promotes mitochondrial fission in preeclampsia. Cell Death Dis 9: 298, 2018.
59. Zhang J, Qu B, Yu W, Zhu Y, Yan X, Shen H and Zhao J: Role of surface ectoderm-specific mitofusin 2 in the corneal morphologic development of mice. Am J Transl Res 11: 3620-3628, 2019.

60. Chapple SJ, Cheng X and Mann GE: Effects of 4-hydroxynonenal on vascular endothelial and smooth muscle cell redox signaling and function in health and disease. Redox Biol 1: 319-331, 2013.

61. Wakabayashi J, Zhang Z, Wakabayashi N, Tamura Y, Fukaya M, Kensler TW, Iijima M and Sesaki H: The dynamin-related GTPase Drp1 is required for embryonic and brain development in mice. J Cell Biol 186: 805-816, 2009.

62. Lorin S, Borges A, Ribeiro Dos Santos L, Souquère S, Pierron G, Ryan KM, Codogno P and Djavaheri-Mergny M: c-Jun NH2-terminal kinase activation is essential for DRAM-dependent induction of autophagy and apoptosis in 2-methoxyestradiol-treated ewing sarcoma cells. Cancer Res 69: 6924-6931, 2009.

63. Nguyen HB, Loomba M, Yang JJ, Jacobsen G, Shah K, Otero RM, Suarez A, Parekh H, Jaehne A and Rivers EP: Early lactate clearance is associated with biomarkers of inflammation, coagulation, apoptosis, organ dysfunction and mortality in severe sepsis and septic shock. J Inflamm (Lond) 7: 6, 2010.

64. Marriott HM, Hellewell PG, Cross SS, Ince PG, Whyte MK and Dockrell DH: Decreased alveolar macrophage apoptosis is associated with increased pulmonary inflammation in a murine model of pneumococcal pneumonia. J Immunol 177: 6480-6488, 2006.

65. Jiang Y, Chen Y and Chen Y: Knockdown of JARID2 inhibits the viability and migration of placenta trophoblast cells in preeclampsia. Mol Med Rep 16: 3594-3599, 20174.

66. Arroyo J, Price M, Straszewski-Chavez S, Torry RJ, Mor G and Torry DS: XIAP protein is induced by placenta growth factor (PLGF) and decreased during preeclampsia in trophoblast cells. Syst Biol Reprod Med 60: 263-273, 2014.

This work is licensed under a Creative Commons Attribution-NonCommercial-NoDerivatives 4.0 International (CC BY-NC-ND 4.0) License. 